\title{
Contemporary Opportunity for Prehabilitation as Part of an Enhanced Recovery after Surgery Pathway in Colorectal Surgery
}

\author{
Zhi Ven Fong, MD, MPH ${ }^{1}$ David C. Chang, MPH, MBA, PhD ${ }^{1}$ Keith D. Lillemoe, MD² \\ Ryan D. Nipp, MD, MPH ${ }^{3}$ Kenneth K. Tanabe, MD ${ }^{2}$ Motaz Qadan, MD, PhD ${ }^{2}$
}

${ }^{1}$ Codman Center for Clinical Effectiveness in Surgery, Massachusetts General Hospital and Harvard Medical School, Boston, Massachusetts

2 Department of Surgery, Massachusetts General Hospital and Harvard Medical School, Boston, Massachusetts

${ }^{3}$ Department of Medicine, Massachusetts General Hospital and Harvard Medical School, Boston, Massachusetts

Address for correspondence Motaz Qadan, MD, PhD, Department of Surgery, Massachusetts General Hospital, 55 Fruit Street, Yawkey 7B, Boston, MA 02114 (e-mail: mqadan@mgh.harvard.edu).

Clin Colon Rectal Surg 2019;32:95-101.

\author{
Abstract \\ Keywords \\ - prehabilitation \\ - optimization \\ - colorectal surgery \\ - surgical oncology \\ - cancer \\ - nutrition \\ - exercise
}

The implementation of upfront, preoperative habilitation ("prehabilitation"), as opposed to postoperative habilitation (rehabilitation), provides a unique opportunity to optimize surgical outcomes, while ensuring that patients receive necessary conditioning that may otherwise be significantly delayed by postoperative complications. In this review, opportunities to design, implement, monitor, and evaluate a surgical prehabilitation program in colorectal surgery are discussed, and broken down to include emotional, physical, and nutritional aspects of care in the preoperative setting.

Traditionally, patients with locally advanced rectal cancer without evidence of distant metastases (stages II and III) undergo chemoradiation to the tumor, followed by surgery, and subsequently adjuvant chemotherapy to best control local and systemic disease associated with the rectal cancer. In addition, there has been an increasing trend in the utilization of neoadjuvant chemotherapy and radiation regimens among patients with other resectable and locally advanced gastrointestinal cancers, such as esophageal, liver, and pancreatic cancers. $^{1-3}$

The hiatus between the time of cancer diagnosis and a scheduled operation provides a unique opportunity for patient optimization, both physically and mentally. ${ }^{4}$ Patient conditioning in this setting is similar to a runner preparing for a marathon. In the weeks preceding the marathon, the runner engages in a rigorous training schedule to enhance athletic performance and optimize physical and psychological endurance. Nutritional optimization constitutes a dynamic component of training, and continues up to race day with implementation of strategies such as "carbohydrate-loading" in the hours preceding the race. Endurance training is challenging and dynamic, and prepares the runner for the physiologic stress of prolonged exercise. ${ }^{5}$ Traditionally, the surgical approach has taken a different view, even though patients embark on a different, yet physiologically similar, insult. Patients are exposed to complex operations, without any requirement for athletic conditioning or exercise, followed ultimately by fasting and dehydration-inducing mechanical bowel preparations in the final hours leading up to the operation. ${ }^{6-8}$

The implementation of upfront, preoperative habilitation ("prehabilitation"), as opposed to postoperative habilitation (rehabilitation), provides a unique opportunity to optimize outcomes, while ensuring that patients receive necessary conditioning that may otherwise be significantly delayed by postoperative complications. In an analogous example, chemotherapy and radiation protocols have been successfully
Issue Theme Enhanced Recovery after Surgery (ERAS) for Colorectal Surgery; Guest Editor: Hiroko Kunitake, MD, MPH
Copyright @ 2019 by Thieme Medical Publishers, Inc., 333 Seventh Avenue, New York, NY 10001, USA. Tel: +1(212) 584-4662.
DOI https://doi.org/ 10.1055/s-0038-1676473. ISSN 1531-0043. 

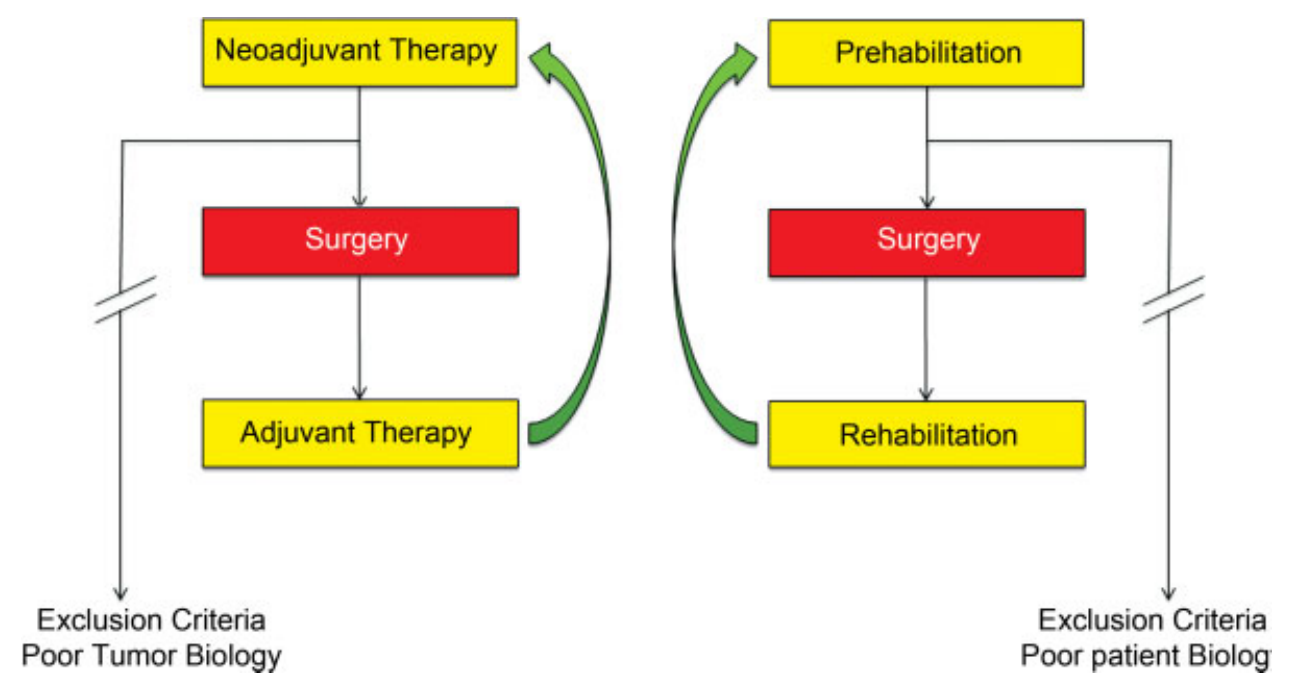

Fig. 1 Comparison of the paradigm shift in the sequence of neoadjuvant therapy administration with the concept of prehabilitation in the surgical oncology patient.

expanded from the traditional adjuvant form to include a neoadjuvant phase prior to an operation ( - Fig. 1). With the implementation of a goal-directed prehabilitation program, perioperative complication rates may decrease and cancerspecific outcomes could potentially be improved. In this era of amplified access to portable technologies and software, in combination with the electronic medical record (EMR), opportunities to closely track performance of patients undergoing intensive prehabilitation are increasingly available.

While some components of prehabilitation and patient optimization are included among enhanced recovery after surgery (ERAS) protocols, the concept of prehabilitation is relatively novel, with paucity of data in this upcoming field. The objective of this manuscript is to discuss opportunities to design, implement, monitor, and evaluate a surgical prehabilitation program in colorectal surgery, which would complement ERAS protocols in improving overall patient outcomes. While prehabilitation may apply to patients with benign colorectal disease in the preoperative phase, a specific emphasis is provided on colorectal cancer in this review.

\section{Rationale for Prehabilitation in Modern Oncologic Care}

Historically, patients underwent surgical resection shortly after receiving the diagnosis of a solid malignancy. However, there has been a steady increase in the median time from diagnosis to operative treatment in recent years, ${ }^{9}$ owing to the deliberate multidisciplinary planning and increasing use of neoadjuvant therapies. ${ }^{10-13}$ Provision of neoadjuvant treatment has provided clinicians with an opportunity to evaluate tumor biology, while ensuring that patients receive systemic therapy. Treatments, when administered in the adjuvant (postoperative) setting, are often delayed or withheld due to surgical complications or patients' inability to tolerate adjuvant treatment after an extensive operation. In addition, a neoadjuvant strategy has been shown to improve resectability of previously inoperable cancers, while redu- cing the complexity of operations secondary when associated with successful tumor shrinkage. Even outside of neoadjuvant treatment protocols, operative management may be deliberately delayed while patients undergo extensive diagnostic workups and multidisciplinary consultations. Any increase in the time interval to operation provides a unique opportunity to maximize patients' preoperative conditioning, to optimize surgical outcomes. ${ }^{14}$

Patient optimization programs in surgery have thus far only been sparsely recognized and explored, with negligibly low uptake. Such programs have been difficult to implement, because they are resource intensive, yet rarely reimbursable. However, the recent shift from fee-for-service to bundled care payments has re-shaped hospital priorities away from maximizing billing for each discrete service to rewarding value of an episode of care. Episode-of-care payments have seen an increase in quality improvement initiative efforts and postdischarge coordination programs, including enhanced recovery programs. ${ }^{15,16}$ These efforts decrease health care costs by reducing postoperative complications and minimizing unnecessary readmissions. ${ }^{17} \mathrm{~A}$ prehabilitation program, therefore, represents a novel opportunity with the potential to contribute toward coordinated, effective, and high-quality care.

The fast pace of contemporary clinical medicine and surgery has placed greater demands on clinicians than ever before. Therefore, the success of a prehabilitation program hinges on how seamlessly it integrates into clinical practice with the least amount of administrative burden. With current advances in EMR systems, there are many technological opportunities that make a modern-day prehabilitation program feasible. Currently at many institutions, EMR systems integrate emergency room, outpatient, and inpatient episodes into a data warehouse. This allows for efficient data collection, automatic tracking of dynamic data, and the immediate availability of point-of-care data via user-friendly interfaces to clinicians at every encounter. Additionally, electronic devices (including smartphones and tablets), which seamlessly integrate into larger EMR systems, provide dynamic data such as steps per 
day, nutritional intake, and calories burnt, with the ability to generate automated goal-directed alerts to users, including patients and physicians, once milestones have been achieved, or missed.

\section{Current Initiatives in Prehabilitation in General Surgery}

The "Strong for Surgery Program" is an initiative developed by the University of Washington's Surgical Care and Outcomes Assessment Program (SCOAP) Comparative Effectiveness Research Translation Network for the state's hospitals. ${ }^{18}$ The aim of the program is to optimize patients' health prior to surgery. In 2015, the American College of Surgeons (ACS) adopted the program and aimed to disseminate the concept to hospitals nationwide. ${ }^{19}$ The program pushes for a checklist of best practices for opioid minimization, preoperative smoking cessation, and glucose homeostasis. ${ }^{18}$ However, the program does not include physical prehabilitation, which is a key component for patients undergoing surgery. In addition, the emphasis was broader, aimed more generally at patients undergoing any surgical intervention. As such, the unique stressor of an oncologic diagnosis and opportunities for intervention remain unaddressed by this protocol.

Another initiative is the Michigan Surgical Home and Optimization Program (MSHOP), which is a statewide program that engages patients undergoing elective inpatient general surgery procedures in the preoperative setting for optimization. ${ }^{20}$ MSHOP provides individualized risk assessment, and empowers patients to regain control of their preoperative conditioning by emphasizing physical activity, smoking cessation, nutritional optimization, and relaxation techniques. Through the early phases of this program, MSHOP has enrolled 500 patients with a $90 \%$ compliance rate. Comparison with matched historical controls demonstrated a reduction of hospital costs by $\$ 2,308$ per patient, and an average length of hospital stay reduction of 2 days. ${ }^{20}$ Once again, specific implementation of such a program in the surgical oncology population has not yet been explored, and effects on cancer outcomes are unknown.

\section{Opportunities for Prehabilitation in Colorectal Surgery}

As with ERAS, colorectal surgery represents an ideal avenue for a prehabilitation program in many ways. First, contemporary neoadjuvant chemotherapy and chemoradiation can be quite toxic, with numerous side effects. In patients undergoing therapy, it is not uncommon that patients require hospitalization for fatigue, dehydration, and nutrition optimization. ${ }^{21,22}$ Preoperative optimization of patients' functional status and physical endurance in that setting might help maximize the chances of completing neoadjuvant treatment without interruption, thereby boosting chances of long-term survival. ${ }^{4,23-25}$

Colorectal cancer incidence increases with age, and the complexity of specialized operations imposes significant physiological stress on older patients, often with increased morbidity and mortality. ${ }^{26-30}$ As a result, elderly patients are less likely to receive life-extending postoperative therapy, such as chemotherapy and radiation, owing to increasing postoperative frailty and associated complications. ${ }^{31-33}$ Data have demonstrated that preoperative conditioning for as short as 2 weeks can be effective in this population. ${ }^{24}$ As such, a 2- to 4-month program if neoadjuvant treatment is contemplated should go a long way to enhancing clinical and cancer outcomes following complex oncologic resections in older patients compared with their younger counterparts.

Finally, patients with newly diagnosed solid tumors often seek long-term survival, an outcome that correlates strongly with response to neoadjuvant and adjuvant therapies, and successful surgical resection without major complications. ${ }^{24}$ A successful prehabilitation program should thus focus on modifiable and actionable predictors of outcomes, and more importantly empower patients to regain some control of their outcomes. Prehabilitation will not only serve to improve the emotional well-being of these patients but may also positively leverage the anxiety and motivation surrounding major oncologic surgery. ${ }^{34}$ Such unique factors are critical for recovery and long-term survival among oncologic patients, particularly in the geriatric oncology population.

\section{Components of a Proposed Prehabilitation Program}

The necessary components of a prehabilitation program would ideally include emotional support, physical therapy, and nutritional optimization (-Fig. 2). Patients should be enrolled in such a program as soon as the diagnosis is established by the multidisciplinary team, and continued through the time of their operation.

The emotional support aspect of the prehabilitation program should begin as soon as possible after the patient's diagnosis. ${ }^{35,36}$ Involvement of patients' family members and caregivers is paramount to the success of the program, since many patients may be overwhelmed with treatment aspects of their diagnosis. In addition, successful implementation of a prehabilitation program would be strictly monitored, which is often aided by patients' social support network. Older patients may be especially vulnerable, as their social support systems may be debilitated (partners), or nonexistent. ${ }^{37,38}$

As part of this proposed prehabilitation framework, patients and their caregivers should be introduced to a patient support group consisting of patients with similar diagnoses who have successfully undergone oncologic resection. The patient support group could be connected via online platforms (e.g., PatientsLikeMe ${ }^{39}$ and WePatients ${ }^{40}$ ) and electronic mail, as well as through weekly or monthly meetings in-person. Connecting patients to survivors can provide hope, improve coping, and reduce the anxiety associated with uncertainty, which ultimately provide motivation to work toward an achievable defined goal. ${ }^{41,42}$

The physical aspect of the proposed program begins with preoperative risk assessment of the patient using tools such as the American College of Surgeons National Surgical Quality Improvement Project (ACS-NSQIP) risk calculator. 


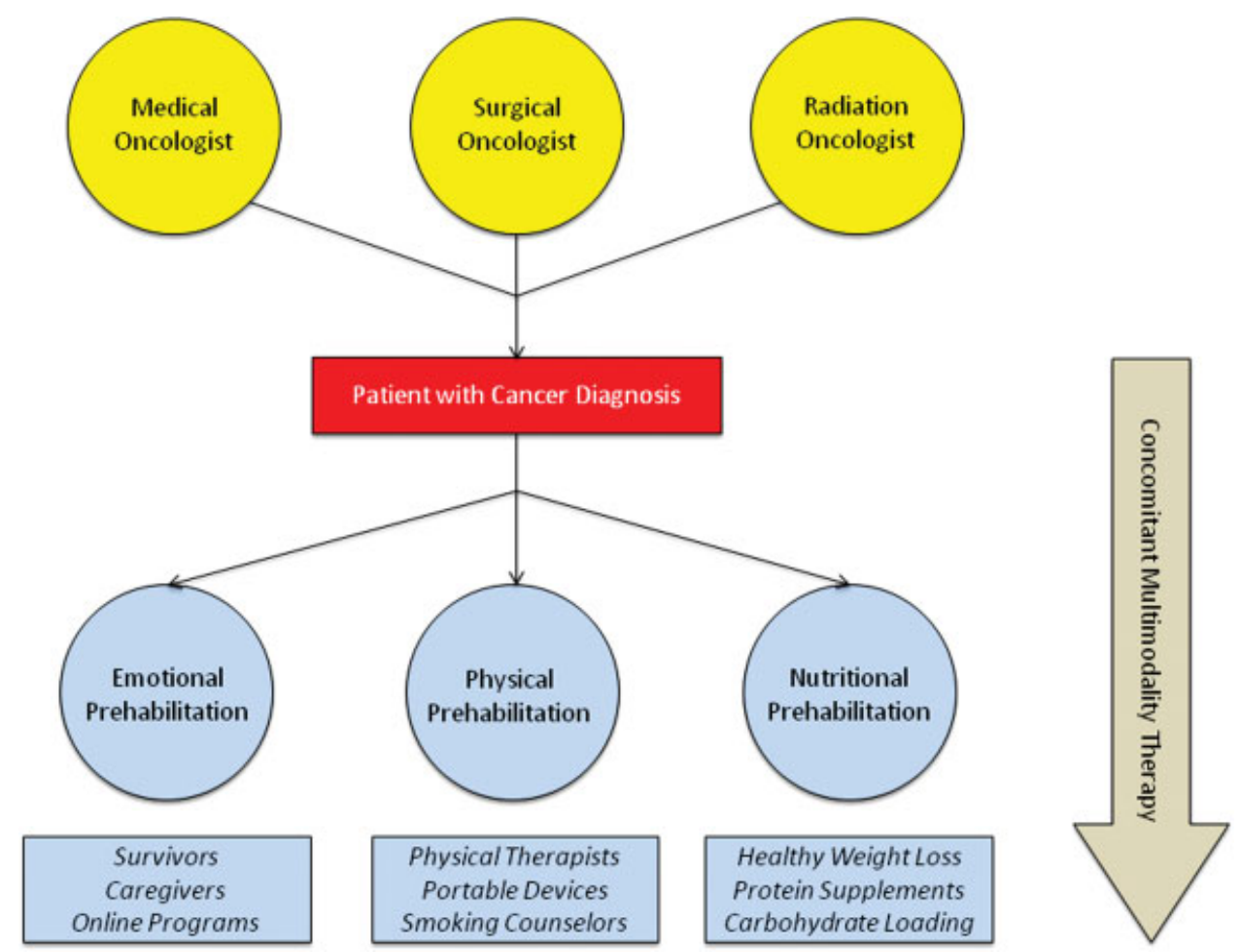

Fig. 2 Proposed prehabilitation program with concomitant multimodality therapy.

Modifiable patient characteristics that contribute to adverse outcomes are identified and shared with the patient and their caregivers. For example, a smoking cessation program and nicotine replacement therapy instituted over a 6 - to 8 -week period can reverse the adverse postoperative outcomes associated with smoking. ${ }^{43}$ A smoking intervention program for patients who are active smokers in the 4 months preceding surgery represents a great opportunity for outcomes improvement, particularly since smoking is a central etiological factor in the development of a majority of solid tumors.

As part of the physical aspect of this proposed program, patients are then enrolled in an exercise program with the goal of increasing their functional capacity to better sustain the physiologic stresses of surgery. Patients may be engaged with a wireless-enabled wearable activity tracker (e.g., Fit Bit) for walking and exercising, with the goal of gradually increasing the number of steps per day. Interestingly, many smartphones also possess equal capability in tracking similar activity (iPhone health app and built-in accelerometer), further highlighting the benefits that smartphones continue to exert within healthcare. An incentive spirometer with a daily tracking flowsheet improves respiratory capacity preoperatively, and, like other elements of physical prehabilitation, allows for seamless continuation postoperatively. ${ }^{44}$ Prehabilitation programs should include exercise regimens that are tailored to provide patients with achievable goals. Patients who are not meeting their targets are notified both remotely and during visits, as are clinicians to their overall progress. Progress is reviewed at every visit, and regular feedback is provided.

Prior studies have evaluated the physical elements of surgical prehabilitation programs. For example, a rando- mized controlled trial in patients with colorectal cancer demonstrated that patients participating in a prehabilitation program consisting of stationary cycling plus weight training prior to surgery were more likely to improve their physical function and recover to their baseline walking capacity postoperatively compared with those who did not participate. ${ }^{25,45}$ A similar trial in patients undergoing cardiac surgery demonstrated not just an improvement in postoperative functional abilities and length of hospital stay but also in quality of life that persisted up to 6 months after the surgery. ${ }^{4}$ In a retrospective analysis of 185 patients undergoing colorectal surgery, patients experienced greater improvement in walking capacity compared with patients in whom rehabilitation started only following surgery. ${ }^{46}$ In a systematic review of five studies encompassing 353 patients undergoing colorectal surgery, prehabilitation protocols have been associated with improved physical capacity. ${ }^{47}$ In the context of colorectal surgery for cancer, the benefits of improved physical tolerance and conditioning may not only improve operative results but also enhance the ability of patients to better tolerate the toxic neoadjuvant and adjuvant therapies they would receive.

In addition to improving emotional well-being and physical conditioning, a nutritional consultation is also sought as early as possible in the preoperative course. Nutritional risk assessment for targeted supplementation and improving postoperative nutrition education are critical elements of any proposed program. In the immediate preoperative setting, patients undergo preoperative carbohydrate-loading, with mandated fasting only up to 2 to 4 hours before surgery. Clear carbohydrate-loading regimens, which are currently 
included in ERAS protocols, permit administration throughout the day prior to surgery, when bowel surgery is anticipated. Preoperative carbohydrate-loading has been shown to decrease postoperative insulin resistance by approximately $50 \%$ compared with overnight fasting, leading to improved surgical outcomes. ${ }^{48,49}$ Randomized controlled trials have demonstrated that preoperative consumption of carbohydrate-containing fluids not only preserves muscle mass after surgery ${ }^{50}$ but also results in earlier return of bowel function and a shorter postoperative hospitalization. ${ }^{49,51,52} \mathrm{~A}$ recent meta-analysis of 21 randomized controlled trials corroborated the findings, demonstrating that preoperative carbohydrate-loading was associated with reduced postoperative insulin resistance and reduced length of hospital stay in patients undergoing major abdominal surgery. ${ }^{53}$ Importantly, deviating away from traditional fasting regimens did not result in any aspiration events during, or after, surgery. ${ }^{49}$

The comprehensive and "bundled" benefits of such a prehabilitation program, including the physical, emotional, and nutritional components outlined earlier, have the potential to yield synergistic benefits in outcomes, similar to the improvements seen with surgical site infection bundles, ${ }^{54,55}$ intensive-care ventilator-associated pneumonia bundles, ${ }^{56}$ central line-associated blood-stream infection bundles, ${ }^{57,58}$ and ERAS bundles. ${ }^{52,59,60}$ The overarching goal of these components is to provide superior short-term and longterm outcomes for patients with cancer who undergo surgical resection of their tumor.

\section{Anticipated Benefit from Prehabilitation in Colorectal Surgery}

As previously mentioned, the field of colorectal surgery stands to benefit significantly from a prehabilitation program, given the increasing time interval from diagnosis to surgery, the high-risk population encountered, the complexity of the surgical intervention, and the accompanying toxicity of therapies necessary to provide long-term survival for patients with cancer. Prehabilitation for these patients may increase their tolerability of neoadjuvant therapies, and maximize the likelihood of treatment completion, including primary resection of the tumor. In addition, decreasing postoperative complication rates increases the likelihood that patients receive postoperative adjuvant treatment where indicated. ${ }^{32,33,61,62}$ These interventions may all translate to improvements in overall and disease-specific survival for patients with colorectal malignancies.

In addition to the potential survival benefits, a prehabilitation program that focuses on patient engagement in their perioperative care has the potential to improve patients' overall satisfaction with care and quality of life. Additionally, improved tolerance of treatment may lead to reduction in emergency room visits and inpatient admissions for treatment toxicity and complications. Reduction in postoperative complications would lead to reduced length of hospital stay and overall surgical readmission rates, which further aligns with patient preferences for their care. ${ }^{63}$

\section{Potential Challenges to Implementation in Colorectal Surgery}

While preliminary data demonstrate that the implementation of prehabilitation in patients undergoing elective general surgery is feasible with encouraging results, ${ }^{20}$ its transferability to the colorectal surgery population is unclear. Patients with cancer are often of advanced age and frailty, and may suffer from inevitable decreased compliance and successful completion rates of any such program. However, this may aid in "selecting out" patients who would not otherwise tolerate a complex surgical procedure, and thus serve as a test of "patient biology," as opposed to "tumor biology." Whether exclusion based on "poor patient biology" will ultimately represent a missed opportunity for resection is unknown. However, avoidance of unnecessary morbidity and mortality is important in patients who may not be able to tolerate an operation. ${ }^{10,33}$

The implementation of a prehabilitation program is not without administrative burden. However, recent advances in EMR can mitigate such burden through automation of a centralized tracking system of patients' prehabilitation program progress. In addition, dedicated assistants employed at various levels in patient care pathways can aid in the absorption of administrative burden by collating patients' progress data points throughout the prehabilitation program. While costs associated with such programs may seem acutely less feasible, the potential savings from the aforementioned benefits would likely offset upfront costs, provided the program is rigorously monitored, implemented, and audited for longterm outcomes and ongoing improvements.

\section{Conclusion}

A prehabilitation program represents a golden opportunity in colorectal surgical oncology that benefits patients, caregivers, and providers alike. It promotes patients as the ultimate stakeholders in their own healthcare, and fosters a collaborative effort between patients and clinicians. Improvements in collective, organized, and systematic care delivery to improve cancer outcomes merit full consideration. Prehabilitation in colorectal surgery, as well as other surgical oncology disciplines, provides one such avenue, among a multitude of new avenues being explored.

Conflict of Interest

None declared.

\section{References}

1 Mirkin KA, Hollenbeak CS, Gusani NJ, et al. Trends in utilization of neoadjuvant therapy and short-term outcomes in resected pancreatic cancer. Am J Surg 2017;214(01):80-88

2 Greenleaf EK, HollenbeakCS, Wong J. Trends in the use and impact of neoadjuvant chemotherapy on perioperative outcomes for resected gastric cancer: evidence from the American College of Surgeons National Cancer Database. Surgery 2016;159(04):1099-1112

3 Bui DL, Yu JB. Trends in adjuvant and neoadjuvant radiotherapy for cancer treatment from 1973 to 2011. Cancer J 2015;21(03): $147-149$ 
4 Arthur HM, Daniels C, McKelvie R, Hirsh J, Rush B. Effect of a preoperative intervention on preoperative and postoperative outcomes in low-risk patients awaiting elective coronary artery bypass graft surgery. A randomized, controlled trial. Ann Intern Med 2000;133(04):253-262

5 Tarnopolsky MA, Atkinson SA, Phillips SM, MacDougall JD. Carbohydrate loading and metabolism during exercise in men and women. J Appl Physiol (1985) 1995;78(04):1360-1368

6 Fa-Si-Oen P, Roumen R, Buitenweg J, et al. Mechanical bowel preparation or not? Outcome of a multicenter, randomized trial in elective open colon surgery. Dis Colon Rectum 2005;48(08): 1509-1516

7 Lavu H, Kennedy EP, Mazo R, et al. Preoperative mechanical bowel preparation does not offer a benefit for patients who undergo pancreaticoduodenectomy. Surgery 2010;148(02):278-284

8 Slim K, Vicaut E, Panis Y, Chipponi J. Meta-analysis of randomized clinical trials of colorectal surgery with or without mechanical bowel preparation. Br J Surg 2004;91(09):1125-1130

9 Bilimoria KY, Ko CY, Tomlinson JS, et al. Wait times for cancer surgery in the United States: trends and predictors of delays. Ann Surg 2011;253(04):779-785

10 Papalezova KT, Tyler DS, Blazer DG III, et al. Does preoperative therapy optimize outcomes in patients with resectable pancreatic cancer? J Surg Oncol 2012;106(01):111-118

11 Katz MH, Wang H, Fleming JB, et al. Long-term survival after multidisciplinary management of resected pancreatic adenocarcinoma. Ann Surg Oncol 2009;16(04):836-847

12 Pawlik TM, Laheru D, Hruban RH, et al; Johns Hopkins Multidisciplinary Pancreas Clinic Team. Evaluating the impact of a single-day multidisciplinary clinic on the management of pancreatic cancer. Ann Surg Oncol 2008;15(08):2081-2088

13 Bilimoria KY, Bentrem DJ, Ko CY, et al. Multimodality therapy for pancreatic cancer in the U.S.: utilization, outcomes, and the effect of hospital volume. Cancer 2007;110(06):1227-1234

14 Gianotti L, Braga M, Nespoli L, Radaelli G, Beneduce A, Di Carlo V. A randomized controlled trial of preoperative oral supplementation with a specialized diet in patients with gastrointestinal cancer. Gastroenterology 2002;122(07):1763-1770

15 Alem N, Rinehart J, Lee B, et al. A case management report: a collaborative perioperative surgical home paradigm and the reduction of total joint arthroplasty readmissions. Perioper Med (Lond) 2016;5:27

16 McMurray A, Johnson P, Wallis M, Patterson E, Griffiths S. General surgical patients' perspectives of the adequacy and appropriateness of discharge planning to facilitate health decision-making at home. J Clin Nurs 2007;16(09):1602-1609

17 Ceppa EP, Pitt HA, Nakeeb A, et al. Reducing readmissions after pancreatectomy: limiting complications and coordinating the care continuum. J Am Coll Surg 2015;221(03):708-716

18 Strong for Surgery, Optimizing Health Prior to Surgery. 2016. Available at: http://www.becertain.org/strong_for_surgery/about. Accessed July 30, 2018

19 Hoyt DB. Looking forward - April 2015 2015. Available at: http:// bulletin.facs.org/2015/04/looking-forward-april-2015/. Accessed July 11, 2016

20 Englesbe MJ, Lussiez AD, Friedman JF, Sullivan JA, Wang SC. Starting a surgical home. Ann Surg 2015;262(06):901-903

21 Dikken C, Sitzia J. Patients' experiences of chemotherapy: sideeffects associated with 5-fluorouracil + folinic acid in the treatment of colorectal cancer. J Clin Nurs 1998;7(04):371-379

22 Marijnen CA, Kapiteijn E, van de Velde CJ, et al; Cooperative Investigators of the Dutch Colorectal Cancer Group. Acute side effects and complications after short-term preoperative radiotherapy combined with total mesorectal excision in primary rectal cancer: report of a multicenter randomized trial. J Clin Oncol 2002;20(03):817-825

23 Jones LW, Peddle CJ, Eves ND, et al. Effects of presurgical exercise training on cardiorespiratory fitness among patients undergoing thoracic surgery for malignant lung lesions. Cancer 2007;110 (03):590-598

24 Valkenet K, van de Port IG, Dronkers JJ, de Vries WR, Lindeman E, Backx FJ. The effects of preoperative exercise therapy on postoperative outcome: a systematic review. Clin Rehabil 2011;25(02):99-111

25 Mayo NE, Feldman L, Scott S, et al. Impact of preoperative change in physical function on postoperative recovery: argument supporting prehabilitation for colorectal surgery. Surgery 2011;150 (03):505-514

26 Makary MA, Winter JM, Cameron JL, et al. Pancreaticoduodenectomy in the very elderly. J Gastrointest Surg 2006;10(03):347-356

27 Bentrem DJ, Cohen ME, Hynes DM, Ko CY, Bilimoria KY. Identification of specific quality improvement opportunities for the elderly undergoing gastrointestinal surgery. Arch Surg 2009;144 (11):1013-1020

28 Riall TS, Reddy DM, Nealon WH, Goodwin JS. The effect of age on short-term outcomes after pancreatic resection: a populationbased study. Ann Surg 2008;248(03):459-467

29 Marrelli D, Roviello F, De Stefano A, et al. Surgical treatment of gastrointestinal carcinomas in octogenarians: risk factors for complications and long-term outcome. Eur J Surg Oncol 2000; 26(04):371-376

30 Louis DJ, Hsu A, Brand MI, Saclarides TJ. Morbidity and mortality in octogenarians and older undergoing major intestinal surgery. Dis Colon Rectum 2009;52(01):59-63

31 Bakens MJ, van der Geest LG, van Putten M, et al; Dutch Pancreatic Cancer Group. The use of adjuvant chemotherapy for pancreatic cancer varies widely between hospitals: a nationwide population-based analysis. Cancer Med 2016;5(10):2825-2831

32 Merkow RP, Bilimoria KY, Tomlinson JS, et al. Postoperative complications reduce adjuvant chemotherapy use in resectable pancreatic cancer. Ann Surg 2014;260(02):372-377

33 Xia BT, Habib DA, Dhar VK, et al. Early recurrence and omission of adjuvant therapy after pancreaticoduodenectomy argue against a surgery-first approach. Ann Surg Oncol 2016;23(13): 4156-4164

34 Tsimopoulou I, Pasquali S, Howard R, et al. Psychological prehabilitation before cancer surgery: a systematic review. Ann Surg Oncol 2015;22(13):4117-4123

35 Silver JK, Baima J. Cancer prehabilitation: an opportunity to decrease treatment-related morbidity, increase cancer treatment options, and improve physical and psychological health outcomes. Am J Phys Med Rehabil 2013;92(08):715-727

36 Silver JK. Cancer prehabilitation and its role in improving health outcomes and reducing health care costs. Semin Oncol Nurs 2015; 31(01):13-30

37 Baider L, Surbone A. Universality of aging: family caregivers for elderly cancer patients. Front Psychol 2014;5:744

38 Verbakel E, Metzelthin SF, Kempen GIJM. Caregiving to Older Adults: Determinants of Informal Caregivers' Subjective Wellbeing and Formal and Informal Support as Alleviating Conditions. J Gerontol B Psychol Sci Soc Sci 2018;73(06):1099-1111

39 Live better, Together!|PatientsLikeMe. Available at: https://www. patientslikeme.com. Accessed November 19, 2016

40 Our Story. WePatients. The Next Generation of Online Healthcare 2016. Available at: http://www.wepatients.com. Accessed November 19, 2016

41 Chiauzzi E, DasMahapatra P, Cochin E, Bunce M, Khoury R, Dave P. Factors in patient empowerment: a survey of an online patient research network. Patient 2016;9(06):511-523

42 DasMahapatra P, Raja P, Gilbert J, Wicks P. Clinical trials from the patient perspective: survey in an online patient community. BMC Health Serv Res 2017;17(01):166

43 Møller AM, Villebro N, Pedersen T, Tønnesen H. Effect of preoperative smoking intervention on postoperative complications: a randomised clinical trial. Lancet 2002;359(9301):114-117

44 Marseu K, Slinger P. Peri-operative pulmonary dysfunction and protection. Anaesthesia 2016;71(Suppl 1):46-50 
45 Carli F, Charlebois P, Stein B, et al. Randomized clinical trial of prehabilitation in colorectal surgery. Br J Surg 2010;97(08):1187-1197

46 Minnella EM, Bousquet-Dion G, Awasthi R, Scheede-Bergdahl C, Carli F. Multimodal prehabilitation improves functional capacity before and after colorectal surgery for cancer: a five-year research experience. Acta Oncol 2017;56(02):295-300

47 Bruns ER, van den Heuvel B, Buskens CJ, et al. The effects of physical prehabilitation in elderly patients undergoing colorectal surgery: a systematic review. Colorectal Dis 2016;18(08):0267-0277

48 Ljungqvist O, Nygren J, Thorell A. Modulation of post-operative insulin resistance by pre-operative carbohydrate loading. Proc Nutr Soc 2002;61(03):329-336

49 Bilku DK, Dennison AR, Hall TC, Metcalfe MS, Garcea G. Role of preoperative carbohydrate loading: a systematic review. Ann R Coll Surg Engl 2014;96(01):15-22

50 Yuill KA, Richardson RA, Davidson HI, Garden OJ, Parks RW. The administration of an oral carbohydrate-containing fluid prior to major elective upper-gastrointestinal surgery preserves skeletal muscle mass postoperatively-a randomised clinical trial. Clin Nutr 2005;24(01):32-37

51 Noblett SE, Watson DS, Huong H, Davison B, Hainsworth PJ, Horgan AF. Pre-operative oral carbohydrate loading in colorectal surgery: a randomized controlled trial. Colorectal Dis 2006;8(07): 563-569

52 Group EC; ERAS Compliance Group. The impact of enhanced recovery protocol compliance on elective colorectal cancer resection: results from an international registry. Ann Surg 2015;261 (06):1153-1159

53 Awad S, Varadhan KK, Ljungqvist O, Lobo DN. A meta-analysis of randomised controlled trials on preoperative oral carbohydrate treatment in elective surgery. Clin Nutr 2013;32(01):34-44

54 Ceppa EP, Pitt HA, House MG, et al. Reducing surgical site infections in hepatopancreatobiliary surgery. HPB 2013;15(05):384-391

55 Anthony T, Murray BW, Sum-Ping JT, et al. Evaluating an evidence-based bundle for preventing surgical site infection: a randomized trial. Arch Surg 2011;146(03):263-269
56 Bird D, Zambuto A, O'Donnell C, et al. Adherence to ventilatorassociated pneumonia bundle and incidence of ventilator-associated pneumonia in the surgical intensive care unit. Arch Surg 2010;145(05):465-470

57 Galpern D, Guerrero A, Tu A, Fahoum B, Wise L. Effectiveness of a central line bundle campaign on line-associated infections in the intensive care unit. Surgery 2008;144(04):492-495, discussion 495

58 Marschall J, Mermel LA, Fakih M, et al; Society for Healthcare Epidemiology of America. Strategies to prevent central lineassociated bloodstream infections in acute care hospitals: 2014 update. Infect Control Hosp Epidemiol 2014;35(07): 753-771

59 Gustafsson UO, Hausel J, Thorell A, Ljungqvist O, Soop M, Nygren J; Enhanced Recovery After Surgery Study Group. Adherence to the enhanced recovery after surgery protocol and outcomes after colorectal cancer surgery. Arch Surg 2011;146(05):571-577

60 Varadhan KK, Neal KR, Dejong CH, Fearon KC, Ljungqvist O, Lobo DN. The enhanced recovery after surgery (ERAS) pathway for patients undergoing major elective open colorectal surgery: a meta-analysis of randomized controlled trials. Clin Nutr 2010;29 (04):434-440

$61 \mathrm{Wu} \mathrm{W}$, He J, Cameron JL, et al. The impact of postoperative complications on the administration of adjuvant therapy following pancreaticoduodenectomy for adenocarcinoma. Ann Surg Oncol 2014;21(09):2873-2881

62 Labori KJ, Katz MH, Tzeng CW, et al. Impact of early disease progression and surgical complications on adjuvant chemotherapy completion rates and survival in patients undergoing the surgery first approach for resectable pancreatic ductal adenocarcinoma - A population-based cohort study. Acta Oncol 2016;55 (03):265-277

63 Moran J, Guinan E, McCormick P, et al. The ability of prehabilitation to influence postoperative outcome after intra-abdominal operation: a systematic review and meta-analysis. Surgery 2016; 160(05):1189-1201 